Arteterapia. Papeles de arteterapia y educación para inclusión social ISSN: $1886-6190$

http://dx.doi.org/10.5209/ARTE.54134

\title{
Arteterapia en una unidad de diálisis pediátrica
}

\author{
Telma Barrantes Fernández $z^{1}$
}

Recibido: 06 de abril de 2016 / Aceptado: 14 de julio de 2016

Resumen. En este artículo se pretende recoger algunos aspectos básicos de la insuficiencia renal, con la intención de comprender y conocer cómo funciona no solo la enfermedad, si no también la unidad de Nefrología y más concretamente la unidad de diálisis y los tratamientos que allí se realizan. De este modo poder dar a conocer a los arteterapeutas esta especialidad, pudiendo facilitar y divulgar el conocimiento que permite una mayor profesionalidad de la disciplina, para su desarrollo y especialización en este contexto.

Palabras clave: Arteterapia; Diálisis; Nefrología;, Insuficiencia Renal.

\section{[en] Art therapy in pediatric dialysis unit}

Abstract. This article covers some basic aspects of renal failure, aiming not only to understand how the illness works but also to explore the nephrology unit and, more specifically, the dialysis unit and the treatments carried out there. Thereby, the present work allows art therapists to learn about this medical specialty. Additionally, it provides and spreads the knowledge necessary for a better professional practice, for development and specialization in this context.

Keywords: Art Therapy; Dialysis; Nephrology; Renal Failure.

Sumario. 1. Introducción; 2. Insuficiencia renal; 3. Tratamiento de diálisis; 4. Complejidad y aportaciones de arteterapia en una unidad de diálisis; 5. Reflexiones a partir de dos casos; 6. Conclusiones; 7. Referencias bibliográficas.

Cómo citar: Barrantes Fernández, T. (2016) Arteterapia en una unidad de diálisis pediátrica, en Arteterapia. Papeles de arteterapia y educación para inclusión social 11, 309-324.

\section{Introducción}

La práctica arteterapéutica con personas que sufren insuficiencia renal o más concretamente durante las sesiones de diálisis es muy escasa. Actualmente en España, no existe ningún tipo de antecedentes bibliográficos. Con ello no se pretende afirmar que no se estén llevando a cabo diferentes proyectos, ya que nos consta que, desde 2011 los alumnos del Máster de Arteterapia y Educación

1 Universidad Autónoma de Madrid

E-mail: telmacc16@gmail.com 
Artística para la Inclusión Social de la Universidad Autónoma y Universidad Complutense, ambas de Madrid, realizan sus prácticas en la unidad de diálisis del Hospital Universitario Materno-Infantil La Paz. El registro de dichas prácticas consta en las memorias que los alumnos han redactado. Aunque se ha tenido acceso a esta documentación, son datos que no se han publicado, pero su lectura ha servido para poder detectar errores, beneficios y complicaciones detectadas a lo largo de los años de prácticas; quedando constancia la gran complejidad de esta unidad a la hora de realizar arteterapia y los interrogantes de los alumnos al abordar las sesiones.

Nos encontramos en Valencia el proyecto CuidArt, que viene realizando intervenciones artísticas desde 2009 en el Hospital de Denia Marina y Salud (proyecto que es apoyado por la aseguradora en salud DKV). Son varias las propuestas que este proyecto plantea para los pacientes, entre ellos el servicio de arteterapia. No se ha podido confirmar que este servicio se realice con pacientes dializados, y mucho menos durante dicho tratamiento. Se puede confirmar que CuidArt tiene un programa conocido como Arte en Vivo, en el que diversos artistas realizan sus creaciones en el propio espacio de las salas de oncología (sala de quimioterapia) y diálisis; e incluso los pacientes pueden participar en dichas creaciones. Las diferentes propuestas artísticas, buscan despertar el interés de los pacientes y focalizar su atención en una alternativa distinta al tratamiento que reciben en las salas de quimioterapia y diálisis. Este proyecto nos hace evidente la cabida que el arte tiene en el medio hospitalario, pero la propuesta que se realiza en la unidad de diálisis no es una referencia propia de arteterapia.

Hay que mencionar por otro lado, que en el Hospital General de Valencia se llevó a cabo en 2014 un proyecto llamado "di sí al día" Fueron los alumnos del Máster de Arteterapia de la Facultad de Bellas Artes de la Universidad Politécnica de Valencia quienes lo pusieron en marcha. Estos alumnos en práctica han ideado una serie de instrumentos para poder ser manipulados por los pacientes, ya que la movilidad de estos se ve perjudicada por las características del propio tratamiento. Al necesitar la conexión a un monitor, es necesario que la zona de acceso que conecta el monitor al paciente se encuentre inmóvil. De este modo, uno de los brazos puede encontrarse impedido de movimiento (este acceso puede ser situado también en la pierna y en pacientes pediátricos, el acceso se coloca en zonas del cuerpo menos móviles). Con estos instrumentos diseñados para pacientes de nefrología, se pretende que ellos puedan expresar sus emociones, motivando un trabajo interno y externo al mismo tiempo. De este modo, se intenta que el proceso de diálisis sea afrontado de una forma llevadera y animada, para que el impacto traumático sea menos destructivo para el paciente.

Como se puede observar, son ya varios los años que la arteterapia y el arte en favor del bienestar del paciente viene abriéndose camino en el campo de la nefrología; pero como ya se ha mencionado, hasta el momento no se han encontrado referencias bibliográficas en España que recojan los resultados de esta especialización. Si buscamos información en otros países como Argentina o Estados Unidos, encontramos diversos estudios que nos reflejan las necesidades, los beneficios y las dificultades de la arteterapia al ser realizada en unidades de diálisis. Nombraremos algunos autores como Weldt (2003), que llevó a cabo una investigación en el Hospital Harlem (New York), en la que usó técnicas de dibujo 
con pacientes sometidos al tratamiento de diálisis. Afirma los beneficios que producen las actividades creativas y significativas para los pacientes. Según Weldt, los procesos artísticos generan en los pacientes actitudes positivas, sentidos de poder, control y libertad, al mismo tiempo que les proporciona un sentimiento de logro. Nishida y Strobino (2005), apoyándose prioritariamente en el estudio de Weldt, entre otros autores, nos presentan un estudio de caso, donde no sólo utilizaron las técnicas de dibujo como hace Weldt, si no que en sus propuestas hicieron uso también de otras técnicas (témpera, acuarela, collage). Nishida y Strobino nos afirman que el arte fue usado por la paciente (su estudio únicamente se basa en el caso de una mujer de 57 años, dializada desde hace 3 años) como una forma de comunicación, aportándole bienestar durante el tiempo de diálisis, a la vez que la sensación del tiempo se le redujo haciendo el tratamiento más llevadero.

En Argentina nos encontramos que en el Hospital Italiano de capital Federal, en el 2007 se realizaron sesiones de arteterapia en la unidad de hemodiálisis pediátrica. Dichas intervenciones fueron presentadas por P. Taboh en el XV Congreso Argentino de Nefrología. Taboh en sus conclusiones, nos indica que el $70 \%$ de los pacientes de la unidad de diálisis participaron en las sesiones de arteterapia; pudiendo observar en los niños, con edades comprendidas entre los 4 y los 15 años, una mejoría en su comunicación, tanto con el equipo médico como entre ellos mismos. Por otro lado, Taboh nos informa que al generar valores psicológicos positivos, ha ayudado a que los niños y niñas acudieran a recibir el tratamiento con una actitud más positiva, disminuyendo así la resistencia al mismo.

Johnson (2008) por su parte nos presenta su estudio Art Therapy and Pediatric Hemodialysis: Creating Therapeutic Space in an Open Unit Medical Setting. En él nos plantea las dificultades y beneficios que surgen al trabajar con niños que están siendo sometidos a diálisis, al mismo tiempo que realizan sesiones de arteterapia. Tras realizar un recorrido por la bibliografía existente sobre nefrología y por su experiencia práctica, concluye que aunque la arteterapia actualmente no es una práctica habitual en las unidades de diálisis, debería ser considerada parte de la intervención terapéutica, siendo integrada en el equipo que atiende a los niños dializados, debido a los beneficios que aporta a los pacientes.

Ross, Hollen, \& Fitzgerald (2006) en su estudio Arts-in-medicine plantean diferentes talleres dirigidos por artistas. Comprobaron que los adultos dializados alcanzaron una mejora en su calidad de vida, teniendo una menor tendencia a la depresión. Afirman que los pacientes asistieron al tratamiento con una voluntad mucho más positiva. Por otro lado, Schreibman (2013) realiza varios planteamientos nuevos en su estudio, plantea la idea de una cultura propia de la unidad. Esto es debido al gran tiempo que los pacientes pasan realizando el tratamiento, por ello el equipo sanitario forma parte de las relaciones sociales de los pacientes. Se crea una conexión muy fuerte entre pacientes y equipo médico (positiva o negativa), generando, de ese modo, entre pacientes y equipo médico, una cultura que les pertenece y construye su propio recorrido histórico.

Esto refleja la importancia emocional que supone el equipo médico para los pacientes, por lo que Schreibman se preocupa por el cuidado de este equipo (enfermeros, dietistas, trabajador social...). Llevó a cabo sesiones enfocadas en la pérdida (fallecimiento de pacientes) y los aspectos emocionales del equipo médico, ya que su trabajo les puede producir un gran desgaste psíquico y merecen ser 
cuidados. Consiguió de este modo, plantear una red social más positiva y saludable, que por consecuencia repercutirá en los pacientes. Este desgaste del equipo sanitario es definido como el síndrome del quemado (burnout), concepto del que también se preocupa Rico (2007) en su investigación. Reflexiona sobre la importancia de cuidar al equipo sanitario, debido al gran desgaste emocional al que están sometidos y la gran cantidad de situaciones estresantes que supone su trabajo.

Con respecto a los pacientes, en el estudio de Schreibman se plantea la arteterapia como un apoyo social, que promueve la expresión verbal y no verbal de los mismos y aumenta la actitud positiva de los pacientes. Todos los autores mencionados en sus estudio incentivan y reflejan la necesidad de continuar investigando.

\section{Insuficiencia renal}

La insuficiencia renal es una enfermedad crónica que afecta al funcionamiento de los riñones. Las guías para pacientes (Conselleria de Sanitat Generalitat Valenciana, Hospital Universitario Reina Sofía) nos indican que el riñón es un órgano vital para el ser humano, teniendo dos funciones principales: una consiste en la limpieza y depuración de sustancias tóxicas y la otra en la eliminación de líquidos mediante la orina. Cuando la insuficiencia renal aparece, significa que una o las dos funciones de las mencionadas, desaparecen o disminuye su efectividad.

Las causas de la insuficiencia renal crónica (IRC) suelen ser múltiples, tiene un carácter irreversible donde el órgano llega a un estado terminal. Se recurre a una terapia sustitutiva o de reemplazo (Diálisis - trasplante), para que el paciente pueda continuar viviendo. Si las circunstancias lo permiten, se recurre al trasplante del órgano, de los cuales hay dos procedimientos de trasplante: de vivos (trasplante planificado) o de cadáver (trasplante repentino). Si no es posible, el paciente pasa por el tratamiento de diálisis (por regla general el equipo médico, intenta retrasar la entrada en diálisis lo máximo posible, por los riesgos que conlleva), que finalizará cuando pueda ser trasplantado.

Se pretende reflejar de forma muy simple algunas cuestiones básicas, debido a que el conocimiento de dichas cuestiones, nos permite hacernos una idea de las vivencias a las que los pacientes se enfrentan. Por otro lado, también nos proporciona una explicación y conciencia de las razones por las cuales es imprescindible adecuar, prevenir y cuidar ciertas acciones en nuestras intervenciones como arteterapeutas durante el tratamiento de diálisis.

\section{Tratamiento de diálisis}

Los riñones de los pacientes con insuficiencia renal, no realizan un correcto filtrado de la sangre, y en algunas ocasiones este filtrado es nulo; lo que supone que las sustancias tóxicas del organismo no son expulsadas. Esta acción necesita ser realizada de forma artificial, encontrándonos de ese modo con el tratamiento de diálisis. 
En la actualidad nos podemos encontrar dos tipos principales de diálisis, una es conocida como Hemodiálisis (HD), que consiste en la limpieza de la sangre, usando un filtro especial llamado riñón artificial o dializador, que se encuentra externo al cuerpo del paciente. El otro tipo es la Diálisis peritoneal (DP), la sangre es limpiada usando un peritoneo, aunque también requiere de monitores externos; la limpieza de la sangre tiene lugar en el abdomen, dentro del cuerpo del paciente. Este tipo de tratamientos por regla general se realizan en hospitales especializados, pero de forma excepcional, en la actualidad, también se puede realizar en el hogar, siempre y cuando las condiciones del paciente lo permitan y el equipo médico lo apruebe.

En las unidades de diálisis, también nos podemos encontrar con pacientes que ya han sido trasplantados y que necesitan de este tratamiento. Esto sucede porque se suelen encontrar inmunodeprimidos y necesitan motivar al órgano, para que finalmente pueda adaptarse al cuerpo del paciente y realizar las funciones renales sin ayuda.

Los pacientes dializados están sometidos a una serie de cirugías menores, para incorporarles el acceso vascular, que permitirá la salida y entrada de la sangre. Hay tres tipos de cirugía para poder acceder a los vasos sanguíneos:

Fístula: es el procedimiento que se utiliza para unir una arteria con una vena, consiguiendo que el flujo sanguíneo sea mayor y la vena se fortalezca; se sitúa por debajo de la piel cerca de la muñeca. Los tubos son gruesos para las venas de los menores y es un procedimiento doloroso para ellos.

- Injerto: consiste en la unión de una arteria y una vena mediante la colocación de un tubo de plástico blando, esto se realiza cuando los vasos sanguíneos no son adecuados. Se suele situar en el brazo o la pierna. Los tubos, al igual que la fístula, son gruesos para las venas de los menores y por lo tanto es doloroso.

- Catéter: es un tubo de plástico estrecho que se introduce en una vena. El catéter suele ser temporal en adultos, pero es el procedimiento más utilizado en pediatría, debido a que los tubos son finos y por lo tanto menos dolorosos. Hay dos tipos de catéter:

- Catéter Agudo, con una duración aproximada de 6 meses a un año, se suele poner a pacientes que van a ser trasplantados de cuerpos vivos, debido a que su intervención puede ser programada y planificada con tiempo.

- Catéter Permanente, cuando la operación no puede ser programada con antelación se coloca este tipo de catéter, que puede durar hasta que llegue el momento del trasplante.

El sobreesfuerzo y los golpes bruscos pueden ocasionar la rotura del mismo y producir hemorragias y fuertes dolores, con la fístula y el injerto sucede igual. Hay casos en los que el catéter ocasiona problemas en los pacientes y no se puede llevar a cabo el tratamiento con normalidad. En esos casos se recurre a un acceso vascular mediante la fístula y si este no surgiera efecto, como última opción se utiliza el injerto. En el caso de los adultos, el orden de preferencia de los accesos vasculares es diferente. 
Cualquiera de los tres procedimientos necesitan de cuidados y vigilancia, ya que se pueden producir hemorragias, infecciones o rotura. Por ello la higiene es algo fundamental en estos pacientes, no sólo para evitar infecciones, sino para prevenir posibles picores, ya que son frecuentes en la insuficiencia renal.

Este acceso es abierto por las enfermeras cuando los pacientes son conectados a la máquina, y vuelve a quedar expuesto a posibles infecciones cuando los pacientes son desconectados; lo que quiere decir, que es un momento en el que toda prevención es poca, limpieza de manos y uso de mascarillas son imprescindibles. $\mathrm{Si}$ todo va bien, los pacientes sólo quedarán expuestos en la conexión y desconexión, pero durante el tratamiento pueden suceder inconveniencias, por lo que las medidas de higiene y protección tienen que estar siempre presente.

El circuito que va de los pacientes al monitor de diálisis, suele permanecer encima de la cama durante el tratamiento. En algunas ocasiones los niños al moverse, pueden sentarse encima "obstaculizando" el recorrido de la sangre, por lo que el monitor comenzará a pitar avisando del fallo. Los movimientos que realizan los niños tienen que ser controlados, porque un exceso de movimiento puede ocasionar el mismo problema que se ha mencionado anteriormente. También hay que tener en cuenta que los pacientes pueden tener dolores por diferentes motivos, pero si tienen algún tipo de vía o injertos en el brazo, la movilización de este puede ser doloroso. Es importante, como ya se ha comentado anteriormente, no motivar el sobreesfuerzo en estas circunstancias debido a que se pueden ocasionar daños o roturas de las vías.

Respecto a la alimentación los pacientes están sometidos a dietas y control de líquidos marcadas por el personal médico; por ello es muy importante no proporcionarles a los niños ningún tipo de sustancia sin previa consulta con su médico o enfermera, porque estaríamos interfiriendo en el tratamiento. A lo largo de las horas de diálisis hay un espacio para la merienda o el almuerzo, esto dependerá de los turnos en los que se realice el tratamiento.

Lo corriente en pacientes de diálisis es que sean sometidos al tratamiento 3 veces semanales durante 4 horas, pero en ocasiones estos pacientes sufren de otras patologías o complicaciones en su tratamiento, pudiendo recibir el tratamiento diariamente y permanecer conectados al monitor de 3 a 5 horas. Por lo que, tanto los días semanales como las horas de conexión varían en cada paciente, dependiendo de las características de cada uno de ellos y de las indicaciones de su médico. Algunos hospitales también actúan como Hospital de día, recibiendo a pacientes que necesitan una única sesión por las circunstancias que sean.

Del conjunto de cuidados, tratamiento médico y psicológico, alimentación e higiene dependerá el nivel de la calidad de vida de los pacientes.

\section{Complejidad y aportaciones de arteterapia en una unidad de diálisis}

A continuación presentamos algunas circunstancias, limitaciones o condicionantes a las que el arteterapeuta se enfrenta al trabajar en este tipo de unidades. Las investigaciones nos indican que es una unidad compleja que necesita ser investigada en la disciplina de la arteterapia. No se pretenden dar o establecer una serie de indicaciones cerradas, más bien se invita a la reflexión continua y al debate 
sobre ciertos elementos como pueden ser el material, sesiones grupales o individuales, cómo insertar/manejar los tiempos y cambios...

La unidad de diálisis tiene una serie de particularidades y características, que a la hora de diseñar las sesiones arteterapéuticas hay que tener muy presentes. Johnson (2008) en su estudio refleja algunas de estas dificultades que también se abordan en este artículo. Por ejemplo las modificaciones constantes de un encuadre cambiante, las "interrupciones" del proceso creador del paciente para realizar las revisiones oportunas por parte del equipo médico, pacientes a los que cambian el turno de su tratamiento, traslados, incorporaciones nuevas o la poca privacidad que puede albergar una sala por la que deambulan constantemente familiares y personal médico. Todas estas cuestiones son circunstancias habituales del ámbito hospitalario. No se pretende hacer un reflejo negativo de estas circunstancias, más bien se invita a la integración de dichas circunstancias en las sesiones arteterapéuticas, dado que forman parte de la cotidianidad de los pacientes. Johnson también nos invita al estudio de dichas circunstancias para que la programación y diseño de las sesiones alcance los objetivos planteados.

Por otro lado, al trabajar en una unidad pediátrica, quiere decir que en una misma sala nos podemos encontrar a niños y niñas de meses de vida hasta 17 ó 18 años. Esto nos indica que el nivel evolutivo de cada uno de los pacientes, puede tener una gran diferencia entre cada uno de ellos. ¿Cómo hacer una sesión individual cuando en la sala hay un grupo de personas que participan de las sesiones de arteterapia? En muchas de las ocasiones, el propio proceso de las sesiones de arteterapia marca estas decisiones. Está claro que aunque cada niño realice acciones completamente diferentes, entre ellos se influencian, intercambian opiniones y comentarios y juegan y bromean con aquel que más cerca tienen. Tenemos que tener en cuenta el hándicap de que los lugares de los pacientes están establecidos y son inmóviles, por lo que el diseño de sesiones grupales en arteterapia en diálisis evoluciona y baila en un término ambiguo entre grupal e individual.

En este sentido, podemos contar que las sesiones que se realizaron durante las prácticas del Máster, se tuvo muy en cuenta este concepto ambiguo entre sesiones grupal e individual. Al principio resulto muy complicado aceptar que no era ni una ni otra, pero una vez que se comenzó a bailar con la ambigüedad y a escuchar las necesidades de los pacientes, la forma de las sesiones surgió sola. En algunas ocasiones se trabajaba por pareja, en otras, compartiendo e intercambiando trabajos y materiales y en otras individualmente. La unión física en esta unidad no es posible, pero el arteterapeuta hace de puente entre una cama y otra y la obra culmina esta unión.

En este artículo, se proporcionan algunas pinceladas generales, tanto del tratamiento como de la insuficiencia renal. Esto nos puede ayudar para hacernos una idea de las circunstancias por las que están pasando los pacientes con los que vamos a trabajar. Tenemos que tener en cuenta que la insuficiencia renal no implica únicamente el tratamiento de diálisis, esto conlleva dietas, controles, medicación, operaciones y espera, una espera continua del órgano que necesitan. Los pacientes dializados conviven durante el tratamiento con otras personas en situaciones similares, esto quiere decir, que ven las complicaciones y las evoluciones de otros pacientes, creando vínculos, preocupaciones, alegrías, 
tristezas y también un recordatorio constante de que los sucesos que les ocurren a sus compañeros también les puede suceder a ellos mismos.

Durante el periodo de prácticas se pudo observar que algunos niños cambiaba su estado de ánimo cuando otro de sus compañeros era trasplantado. Aquellos que llevaban más tiempo en tratamiento les afectaba ver que tenían que continuar esperando un órgano, mientras que sus compañeros más nuevos eran trasplantados. Al mismo tiempo que se alegraban por la mejoría de su amigo, estas emociones contradictorias entre sí producían en el niño un estado de confusión y malestar. Por otro lado, se observaba también un estado de preocupación general cuando algún compañero era ingresado de forma permanente en el hospital. La compasión, las dudas, la necesidad de hablar sobre las razones del ingreso y la empatía se agudizaban.

Como ya hemos visto, para este tratamiento es necesario un acceso vascular. En el caso de los niños, suelen incorporarse en lugares del cuerpo que no impliquen un exceso de movimiento. Los adultos suelen tener ese acceso en el brazo o pierna, esto es importante, ya que como se ha mencionado anteriormente un sobreesfuerzo dañaría al paciente.

¿Cómo solventar este problema? Los alumnos del Máster de Arteterapia de la Facultad de Bellas Artes de la Universidad Politécnica de Valencia, diseñaron una serie de piezas que facilitaban a los pacientes el movimiento y evitaban el riesgo de las "manchas" que suponen las técnicas plásticas. Weldt (2003) soluciona esta problemática con la técnica del dibujo. Considera que es más higiénica y dado que los pacientes de su estudio tenían el acceso vascular en el brazo izquierdo, no tenían problema de movilidad para esta técnica. Nishida y Strobino (2005), por el contrario, deciden incorporar las técnicas húmedas y consideran que siempre y cuando la higiene permanezca presente, no tiene que verse como una amenaza. Consideran que la limitación de técnicas limitan al paciente en su creación y esto podrían no ser beneficioso en su proceso de expresión. Hay que mencionar que el tratamiento de diálisis es un circuito totalmente cerrado, por lo que los materiales en caso de derrame no pondrían en peligro al paciente. Es importante una higiene impoluta, por el hecho de que la desconexión se produce en la cama o sillón en el que el paciente se encuentra en las sesiones de arteterapia. Este momento en el que el acceso vascular se abre para desenganchar el monitor, es un momento clave, es justamente en este instante donde podrían ocasionarse las infecciones. Por otro lado, hay que tener presente que en caso de urgencia, el paciente sería intervenido de inmediato. Si el paciente o la cama se encuentra "manchado" de pintura o cualquier otro material, antes de intervenirlo habría que limpiar todo; esto ocasionaría retrasos en la intervención médica y por lo tanto se pondría en riesgo al paciente.

Las soluciones que encontramos durante el periodo de práctica fueron muy sencillas pero muy útiles. Cuando se utilizaba tempera o escayola se cubría la mesa de trabajo (y si se veía oportuno también las piernas del paciente) con mantas empapadoras. Tener toallitas al alcance de la mano permite limpiar con rapidez y de forma muy simple. En algunas ocasiones también se les proporcionaron guantes a los pacientes para que trabajaran con ellos puestos, de este modo las manos siempre permanecen limpias; esto es importante porque se puede correr el riesgo de que los más pequeños se toquen el acceso bascular con las manos manchadas (este 
permanece siempre tapado con gasas y apósitos y pueden ser cambiados en cualquier momento, pero se recomienda total prevención).

Con respecto a los beneficios de las intervenciones de arteterapia al realizarse durante el tratamiento de diálisis, Weldt (2003) afirma como ya se ha mencionado, que el proceso artístico genera aptitudes positivas. En este sentido se puede mencionar y confirmar que las enfermeras que fueron entrevistadas durante el periodo de prácticas, afirmaban el cambio de aptitud de los niños durante los días que se realizaban las sesiones de arteterapia. Se encontraban mucho más animados y durante el tratamiento permanecían despiertos, ya que el resto de días solían permanecer dormidos o más decaídos. Estos datos meramente son una observación de las prácticas. Actualmente se está llevando a cabo una investigación al respecto para confirmar y verificar de forma más relevante este tipo de observaciones. Tanto Schreibman, Nishida y Strobino en sus diferentes investigaciones, nos afirman la actitud positiva y el cambio de ánimo en los pacientes cuando realizan arteterapia.

Estos cuatro autores que se están mencionando, también afirman que los participantes en las sesiones experimentan que el tiempo de tratamiento es menor. El tiempo en realidaz no sufre variación, pero para ellos es muy importante que esas cinco horas pasen lo más rápido posible. Este punto también pudo ser observado durante las prácticas, debido a que era frecuente que los niños se asombraran cuando se finalizaban las dos horas de intervención arteterapéutica.

\section{Reflexiones a partir de dos casos}

Dos alumnas del Máster de Arteterapia y Educación Artística para la Inclusión Social, de las Universidades Complutense y Autónoma de Madrid; llevamos acabo durante el mes Octubre de 2014 hasta Mayo de 2015 en el Hospital Universitario Materno-Infantil La Paz en Madrid las prácticas en la unidad de diálisis pediátrica, de las cuales ha surgido este artículo.

Se llevaron a cabo unas 22 sesiones, estas tenían lugar los sábados por la mañana, de $10 \mathrm{~h}$. a $12 \mathrm{~h}$., dado que el resto de días tenían clases con el profesor del hospital y los sábados no tienen actividades programadas, excepto las visitas de los voluntarios. Todas las intervenciones tenían una duración de dos horas con una parada a mitad de sesión, para que los niños pudieran tomar el almuerzo; por lo que hay que retirar todo el material y dejar sitio a las bandejas de comidas.

En dichas sesiones se pusieron de manifiesto una serie de circunstancias y características que incentivaron la creación de este artículo, y de una pequeña investigación para profundizar en las cuestiones que aquí nos ocupan. A continuación reflejaremos algunos de los resultados y reflexiones, haciendo evidente la necesidad de esta disciplina en las unidades de diálisis. No se pretende reflejar un estudio de caso concreto, únicamente se pretende mostrar algunos de los sucesos más relevantes que ocurrieron en el transcurso de dichas sesiones. La finalidad es mostrar el valor de este tipo de intervenciones para los pacientes que están sometidos al tratamiento de diálisis.

El nene (niño de 3 años) 


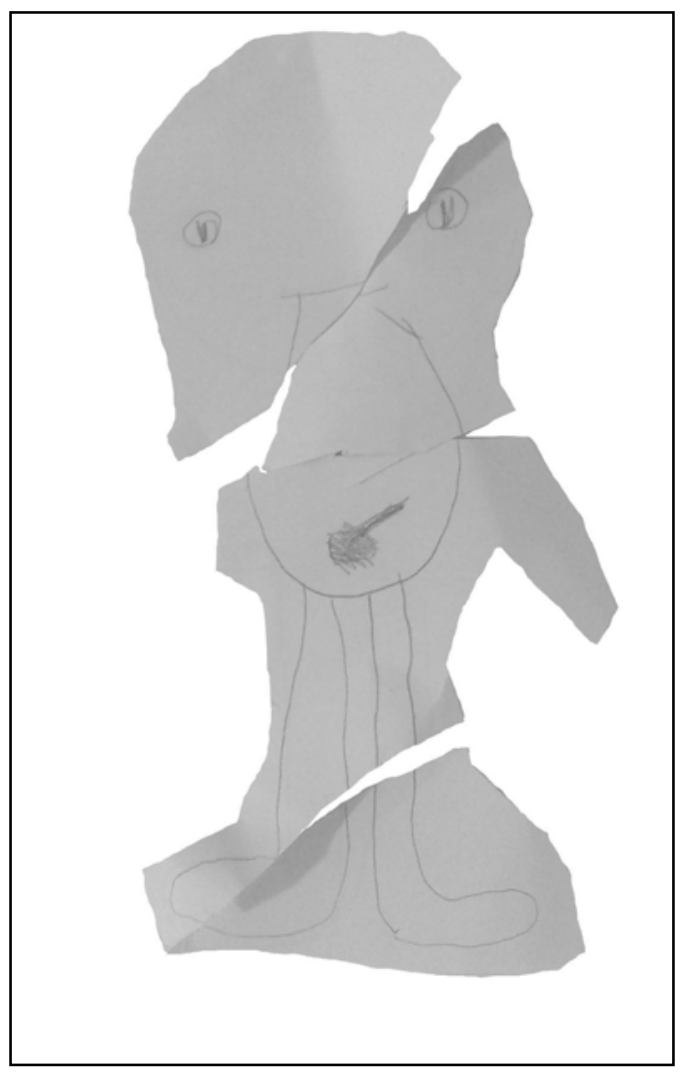

Figura 1: Figura humana de un niño

Podemos observar una figura humana de un niño fragmentada, todo el proceso creativo del dibujo fue un proceso de evolución, maduración y destrucción, una destrucción que no fue entendida como un rechazo o inconformismo de la obra, una destrucción que no invita a la desaparición total, si no un final que abre y crea otra forma de ser y de estar, un final del propio proceso que a su vez crea varias formas que proceden de la misma.

En el proceso, el niño fue capaz de exteriorizar cómo percibía su cuerpo, qué sentido le daba a cada una de las máquinas que le rodeaban, qué tipo de relación mantenía con ellas, cuál adquiría más valor y lo importante que era para él que el botón de la barriga del dibujo estuviera cerrado. Niño y dibujo se funden en uno solo, ambos se comunican, ambos necesitan de cuidados al mismo tiempo que se convierten en cuidadores, en protectores. Agitación y miedo se hicieron presente durante el proceso de creación, pero también tranquilidad, control y determinación, un proceso de decisiones constantes que el niño fue capaz de afrontar, a través de la proyección de sí mismo en el dibujo y el acompañamiento de la arteterapeuta.

Es importante que este tipo de dinámicas tengan lugar, que la libertad de expresión y comunicación se les acerque a los pacientes. Profundizar en cómo les afecta el tratamiento, qué les preocupa, qué temen, qué piensan, acompañarles en la toma de conciencia de sus emociones. Relajar las tensiones que les pueda ocasionar 
durante esas 3 ó 5 horas en las que permanecen conectados a su monitor, qué les condiciona en sus movimientos, en su día a día. Sostener el impacto que la enfermedad y su tratamiento conlleva, la familiarización con el hospital y los materiales que le rodean. Son cuestiones que necesitan tener el lugar que les corresponde, son cuestiones que necesitan ser contadas, expresadas, sentidas, escuchadas, son cuestiones que necesitan ser valoradas. Los pacientes se enfrentan a una incertidumbre constante, a una espera muy pesada y a menudo larga. Un cambio de su vida cotidiana muy brusco, por lo que resulta fundamental que el bienestar psíquico sea cuidado, protegido y es primordial sostenerles en todo el proceso de la enfermedad a la que se enfrentan día tras día.

Con este dibujo y durante el proceso de creación, podemos ver que el impacto del tratamiento es algo que el niño vive y siente, por lo que el escuchar y cuidar ese sentir del niño, es fundamental, ya que de esto depende su actitud ante el tratamiento y la enfermedad.

\section{Jugando al fútbol en diálisis (niño de 16 años)}

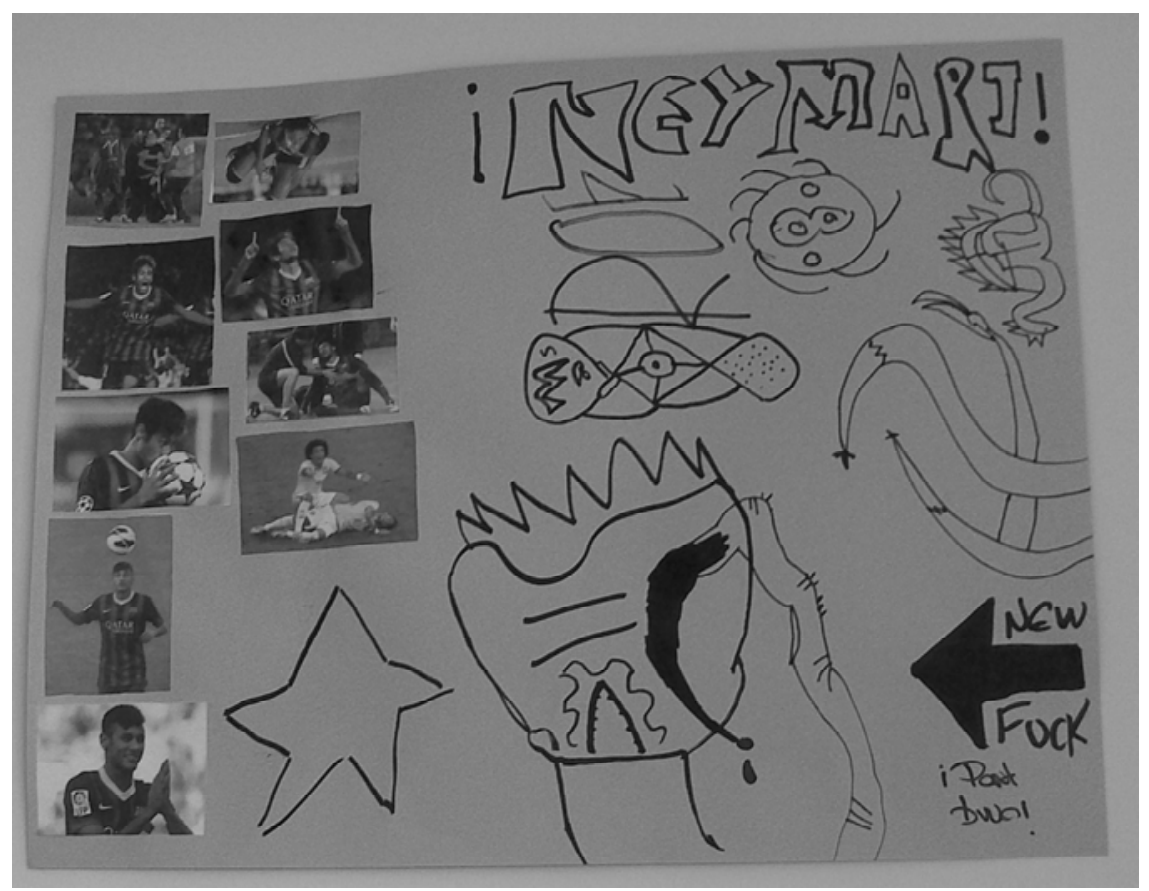

Figura 2: Cartel de Neymar 


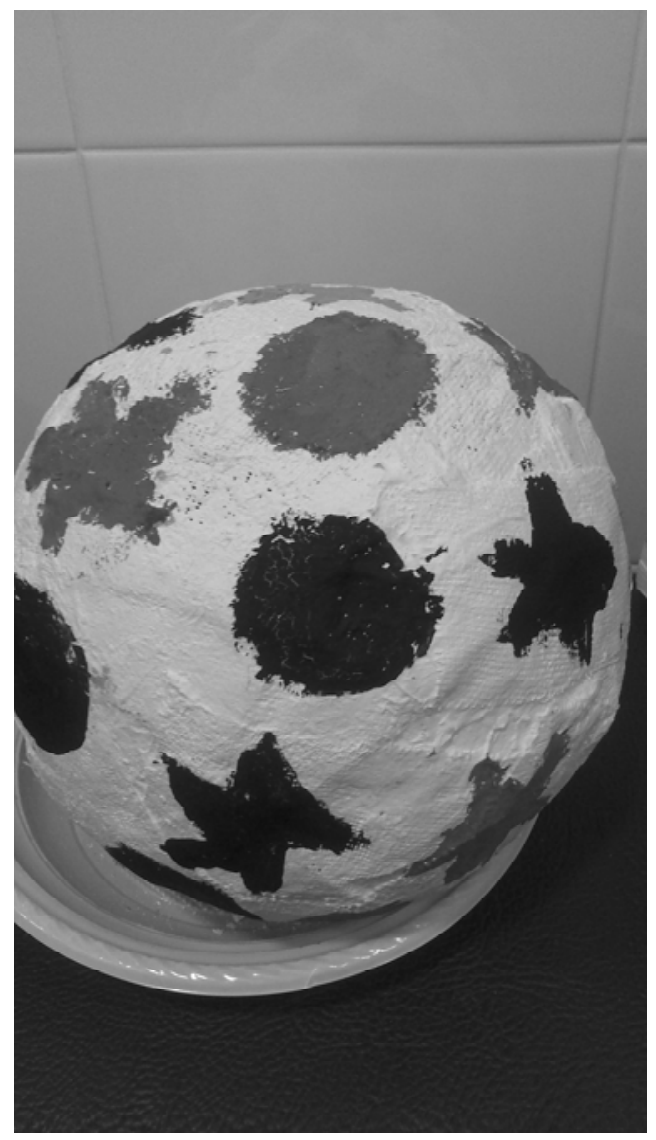

Figura 3: Balón de fútbol en escayola

Es obvio que no se puede jugar al fútbol en una unidad de diálisis, mientras que los niños permanecen conectados a sus correspondientes monitores, pero sí se puede jugar si el juego se mira desde otro punto de vista. Si transformamos esa cancha de fútbol en una cartulina, si transformamos el balón, la portería, si cambiamos las reglas del juego y dejamos libre la imaginación, entonces en una unidad de diálisis puede jugarse a todo.

En las imágenes que aquí presentamos podemos ver cómo este niño de 16 años; como a otros muchos niños de su edad, le interesa este deporte, lo sigue y practica. Esto implica que este deporte los identifica (equipo de futbol), los sociabiliza (juego en grupo) les sirve para descargar tensiones, les marca objetivos (ganar el partido), le muestra la importancia de una estructura, a ser constantes (normas de juego), entre otras muchas cuestiones.

¿Qué ocurre si impedimos jugar a un niño de 16 años cuando el fútbol es algo muy importante para él?. Obviamente el enfado y la rabia se harán presente, en este caso no podíamos ignorar el valor que el niño le daba a este deporte. Es algo que él mostraba en cada una de las sesiones y en cada una, se presentaba de una forma diferente. La preocupación por las limitaciones físicas, las lamentaciones por 
no poder jugar con sus amigos, debido a la imposición de las sesiones de diálisis o el temor de romper el catéter durante el juego. Como no podía ser de otra manera, se le dio su lugar, un lugar donde el niño controlaba y se enfrentaba a todo lo que allí ocurría. Unas veces son preocupaciones, otras temores, otras ilusiones y sueños, en otras ocasiones la frustración, la rabia y el desconcierto se hacían patentes. El fútbol, los jugadores, el balón, pudieran ser las palabras que este niño de 16 años necesitaba para hablar de él. Es importante hablar de uno mismo y hablar con uno mismo para conocerse. Este niño hablaba de él en todos los sentidos, de él y su enfermedad, de él y sus amigos, de él y su futuro, y esto nos llevó a que semana tras semana el fútbol no fuera imprescindible para expresar lo que sentía. Evolucionó su proceso de creación encontrando nuevos lenguajes que le ayudaron a contar su historia de otras formas.

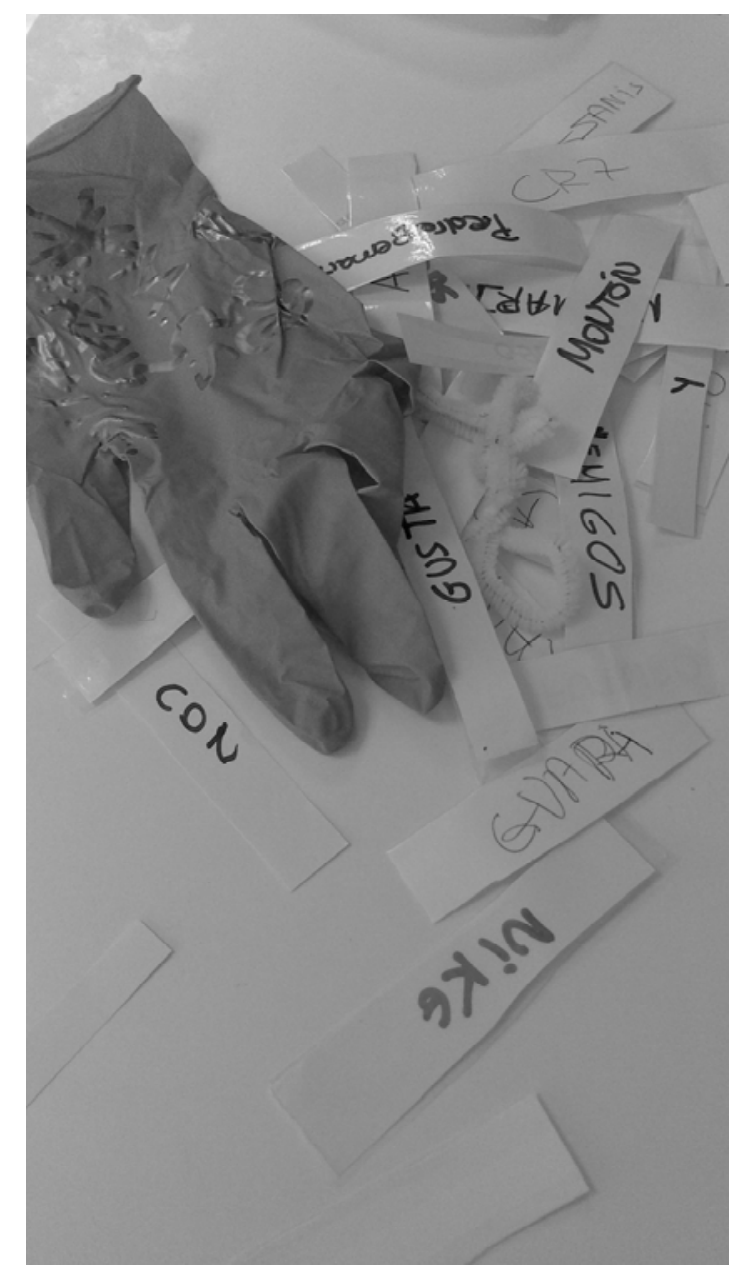

Figura 4: Palabras y guante 


\section{Conclusiones}

En primer lugar, como hemos podido observar, las circunstancias que se dan en una unidad de diálisis son un tanto particulares, y a la hora de realizar arteterapia es importante sopesar y conocer dichas particularidades. Aunque no se ha profundizado en esta cuestión en exceso, hay un detalle que por el momento podemos confirmar, y es que, durante el momento de la conexión y desconexión de los pacientes al monitor de diálisis, los arteterapeutas no podemos estar presentes, dado que es el momento de mayor riesgo (infecciones) y los protocolos hospitalarios, como corresponde, son muy rígidos. Por lo tanto, las sesiones de arteterapia tienen que dar lugar cuando el personal médico permita la entrada a la sala y finalizarán antes de la desconexión de los pacientes. Por otro lado, al igual que plantea Johnson (2008) en ese margen que tenemos para actuar, el servicio médico es prioritario. Por lo tanto las revisiones y controles del tratamiento es un acto cotidiano, que deben formar parte de las intervenciones con total naturalidad. Tenemos que tener en cuenta que las sesiones de arteterapia en este tipo de unidades, podrán ser "interrumpidas" en varias ocasiones, con lo que supone que el paciente pueda llegar a vivir estas interrupciones con molestias, por lo que tendremos que valorar cómo manejar esta circunstancias.

El conocer las medidas protocolarias del hospital, el conocimiento de la enfermedad, los posibles riesgos del tratamiento, nos podrían facilitar el trato y la comprensión de los pacientes, al mismo tiempo que se facilita el trabajo entre equipos (equipo médico, arteterapeuta, psicólogos...). Podríamos mencionar más detalladamente y por poner un ejemplo, las medidas higiénicas; estas son esenciales a la hora de trabajar en hospitales, para evitar contagios e infecciones. El conocer estas medidas, sus riesgos, los protocolos de limpieza de manos, la limpieza de materiales que se pasan de paciente a paciente, las medidas y razones de aislamiento, nos evita cometer errores.

Por otro lado, como arteterapeutas, nos vemos en la tesitura de valorar qué materiales usar y cuáles no, cuales son "más higiénicos" y cuales es necesario prescindir de ellos. En un principio como se ha mencionado, el tratamiento tiene lugar dentro de un circuito cerrado, por lo que no hay riesgos de que la sangre del paciente se contamine por la posible "suciedad" de los materiales. Aun así, es imprescindible tomar ciertas precauciones como no "manchar" en exceso, buscar medios para conservar la higiene del lugar y del paciente, por supuesto, el acceso vascular tiene que estar totalmente protegido. Estas valoraciones, los procedimientos y qué materiales usar, siempre es un tanto relativo y será el arteterapeuta el que debe valorar y pactar con sus pacientes, ya que son estos los primeros en preocuparse por una higiene impoluta. Como ya hemos podido ver, son varios los autores que han reflejado su preocupación por este punto, y las propuestas ante esta cuestión también han resultado ser variadas, por ejemplo; Nishida y Strobino (2005) trabajan con diferentes materiales en las sesiones de arteterapia, dado que consideran que se limita la creación de los pacientes si únicamente le proporcionamos una sola técnica. Weldt como ya se mencionó, decide no arriesgar y trabaja únicamente con el dibujo, esto nos indica que los tres autores se embarcaron en una reflexión y toma de decisiones a la hora de diseñar sus sesiones, por lo que creo que esta reflexión es una constante permanente y (que 
las sesiones sobre) los materiales que usar o no varían en cada caso. Tras este primer contacto en la unidad de diálisis, considero y basándome en la idea de Nishida y Strobino (2005), que alternativas como proteger la zona de trabajo con empapadores, que los pacientes trabajen con guantes en las manos, tener al alcance toallitas o el uso de la herramientas digitales; son detalles que si se tienen en cuenta, nos pueden facilitar el mantenimiento de la higiene sin desechar ciertas técnicas artísticas.

Por otro lado, todos los autores (Weldt; 2003, Johnson; 2008, Schreibman; 2013, Taboh; 2007, Nishida y Strobino; 2005, Ross, Hollen y Fitzgerald; 2006) que se han mencionado anteriormente, coinciden que las sesiones de arteterapia proporcionan a los pacientes diferentes beneficios como: una actitud más positiva frente a la situación que enfrentan, disminuye la resistencia que puedan sentir ante el tratamiento, refuerzan el sentimiento de poder, control y libertad de los pacientes, al mismo tiempo que aumenta la comunicación y les reduce la sensación del tiempo del tratamiento de diálisis.

Todos los autores que han trabajado en esta línea, insisten en la necesidad de continuar investigando. Es obvio que hasta el momento son escasos los datos encontrados, por ello las conclusiones realizadas se basan en la propia experiencia de práctica para finalizar el Máster de Arteterapia y Educación Artística para la Inclusión Social, el trabajo de final de máster, en el que se realizó una pequeña investigación sobre estas cuestiones y junto con la comparación con las investigaciones halladas. Por ello se continua investigando. Esto no nos hace abarcar toda la problemática existente, aún quedan muchas preguntas por resolver y la necesidad de registrar los beneficios que aportan las sesiones de arteterapia a los pacientes dializados, para así demostrar dichos resultados. En este artículo, únicamente se ha podido realizar unas pequeñas pinceladas, que nos pueden ayudar a comprender y trabajar con los pacientes que tienen insuficiencia renal y están sometidos a un tratamiento de diálisis.

\section{Referencias bibliográficas}

Alonso, A. y Fijo, J. (2014) Hemodiálisis pediátrica. Protocolos Hemodiálisis pediátrica. 3(25) pp. 403-420 www.aeped.es.

Generalitat Valenciana Conselleria DE Sanitat. Información al Paciente en Hemodiálisis. Hospital del Vinalopo.

Johnson, A (2008) Art Therapy and Pediatric Hemodialysis: Creating Therapeutic Space in an Open Unit Medical Setting. Canada. Concordia University.

Malchiodi, C. A. (1999) Medical Art Therapy with Children. London. Jessica Kingsley Publishers.

Nishida, M. \& Strobino, J. (2005) Art Therapy with a Hemodialysis Patient: A Case Analysis. Art Therapy: Journal of the AATA. 22(4) pp. 221-226.

Petroff, T. (2004) A contribução da utilizaão dos recursos artísticos e lúdicos pelo psicólogo hospitalar no tratamento de pacientes renais no Hospital do Rim e Hipertensão. Brasil. Pontificia Universidade Católica de São Paulo. 
Ross, A. Hollen, T. \& Fitzgeral, B. (2006) Observational study of an arts-in-medicine program in an outpatient hemodialysis unit. American Journal of Kidney Disease, 47(3), 462-468.

Schreibman, R. C. (2013). Art Therapy and Hemodialysis Coping Cratively With Kidney Failure. En Malchiodi, C. A. (Ed.), Art Therapy and Health Care. (pp. 212-224). London. The Guilford Press.

Servicio de Nefrología, Guía para pacientes con tratamiento de hemodiálisis. Hospital Universitario Reina Sofía.

Taboh, P. (2007) Arteterapia con pacientes pediátricos durante la sesión de hemodiálisis. XV Congreso Argentino de Nefrología. 5(2) p. 141.

Venado, A. Moreno, J. Rodríguez, M. y López, M. (2009) Insuficiencia Renal Crónica. Unidad de proyectos especiales Universidad Nacional Autónoma de México.

Ventura et al. A. (2011) CuidArt: Proyecto de Arte del Departamento de Salud de Dénia. Arte, Individuo y Sociedad. 23 (Especial) pp. 165-180.

Weldt, C. (2003) Patients' Responses to a Drawing Exprerience Hemodialysis unit: A Step Towards Healing. Art Therapy: Journal of the AATA. 20(2) pp. 92-99. 